\title{
Human-centric dialog training via offline reinforcement learning
}

\section{Natasha Jaques*12, Judy Hanwen Shen**1, Asma Ghandeharioun ${ }^{1}$, Craig Ferguson ${ }^{1}$, Agata Lapedriza $^{1}$, Noah Jones ${ }^{1}$, Shixiang Shane $\mathbf{G u}^{2}$, Rosalind Picard ${ }^{1}$}

\author{
*Equal contribution \\ ${ }^{1}$ Massachusetts Institute of Technology, Cambridge, USA \\ <judyshen, asma_gh, agata, ncjones, roz>@mit.edu \\ ${ }^{2}$ Google Research, Mountain View, USA \\ $<$ natashajaques, shanegu>dgoogle.com
}

\begin{abstract}
How can we train a dialog model to produce better conversations by learning from human feedback, without the risk of humans teaching it harmful chat behaviors? We start by hosting models online, and gather human feedback from real-time, open-ended conversations, which we then use to train and improve the models using offline reinforcement learning (RL). We identify implicit conversational cues including language similarity, elicitation of laughter, sentiment, and more, which indicate positive human feedback, and embed these in multiple reward functions. A wellknown challenge is that learning an RL policy in an offline setting usually fails due to the lack of ability to explore and the tendency to make over-optimistic estimates of future reward. These problems become even harder when using RL for language models, which can easily have a 20,000 action vocabulary and many possible reward functions. We solve the challenge by developing a novel class of offline RL algorithms. These algorithms use KL-control to penalize divergence from a pretrained prior language model, and use a new strategy to make the algorithm pessimistic, instead of optimistic, in the face of uncertainty. We test the resulting dialog model with ratings from 80 users in an open-domain setting and find it achieves significant improvements over existing deep offline RL approaches. The novel offline RL method is viable for improving any existing generative dialog model using a static dataset of human feedback.
\end{abstract}

\section{Introduction}

Training open-domain dialog models is inherently difficult, since for each utterance there are many acceptable responses, yet no perfect response. While supervised learning from conversational corpora allows models to learn grammatical structure and even topic coherence, these models do not gener- alize, since the training objectives mostly lead the models to memorize responses within the corpus.

Humans are the ultimate authority in evaluating what makes one conversational reply better than another. To learn from real conversations with humans, we created an interactive, online platform which hosted a diverse set of neural network dialog models that users could chat with in real time. However, when learning from human interactions in the wild it is crucial to be able to learn offline and test the policy before deploying it, lest it learn inappropriate behaviors (e.g. Horton (2016)). Thus, we need to train and test models offline, to ensure safe model outputs. In order to safely learn to optimize human feedback we pursued an offline reinforcement learning approach to training dialog models (see Figure 1).

Offline RL is challenging; most deep RL algorithms fail to learn from data that is not heavily correlated with the current policy (Fujimoto et al., 2018). Even models based on off-policy algorithms like $Q$-learning fail to learn in the offline RL setting, as the model is not able to explore. If the offline dataset is not sufficient to cover the input-response space, offline RL models suffer from extrapolation error, learning arbitrarily bad estimates of the value of responses not contained in the data.

We solve these problems by developing a new method for offline RL. The method starts by leveraging a pre-trained language model to constrain offline RL updates. While training with RL, we penalize divergence from this prior model using forms of KL-control. This combats extrapolation error, and ensures that the RL model learns a policy that stays close to the distribution of realistic language, while learning to maximize positive human responses using the offline data. Further, we use dropout to obtain uncertainty estimates of the target $Q$-values, and to obtain a lower bound to alleviate over-optimistic bias in estimating future 
reward. We show that this new method is able to learn successfully from many different reward functions, even in a very large space with 20,000 tokens.

Both linguistic theory (e.g. Grice's Maxims (Grice, 1975)) and empirical experiments correlating human judgement with language features suggest that there are many criteria that could be used to evaluate a conversational agent (Ghandeharioun et al., 2019; Adiwardana et al., 2020). We develop a set of reward functions for our dialog agents to optimize, which are designed to approximate implicit human preferences expressed during conversational responses. We show that the new method is better able to optimize these rewards using the offline data, and when tested with a new set of 80 human conversation partners, leads to more positive responses and higher quality ratings than a state-of-the-art offline deep RL method.

Novel contributions of this paper are:

- A new offline RL method, Way Off-Policy (WOP) learning, which introduces the use of KL-control from a pre-trained model to reduce extrapolation error, and an approach to make estimates more pessimistic in the face of uncertainty.

- Experiments showing the effectiveness of WOP above strong offline RL baselines.

- An investigation into developing conversation rewards based on how human preferences are implicitly expressed in text. We are the first work to learn from implicit signals in conversation using offline RL.

\section{Related Work}

\subsection{Dialog}

Improving dialog systems with RL has largely been restricted to task-oriented dialog systems, which have a limited number of task-specific actions (Fatemi et al., 2016; Gašić et al., 2011; Liu and Lane, 2017; Liu et al., 2018; Su et al., 2017). Some of these approaches incorporate human input through explicit, manual feedback (Shah et al., 2018) or implicit signals (e.g. the user interrupting the system or starting over) (Shi and Yu, 2018).

$\mathrm{RL}$ in the open-domain dialog setting is less explored (Li et al., 2016, 2017b, 2018). Authors may choose to use a highly restricted action space; for example, using RL to choose which dialog model to

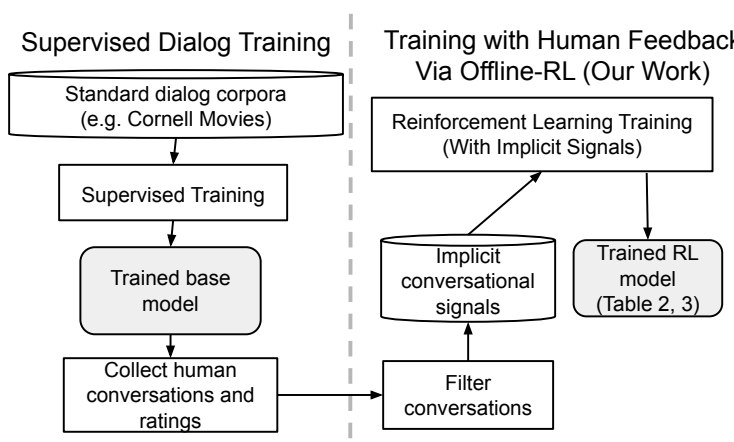

Figure 1: Schematic diagram of our method for training with human conversation cues via offline RL. Unlike traditional approaches which stop at using explicit feedback to evaluate static conversations, we allow humans to freely interact with dialog models, and compute metrics based on their implicit satisfaction which are optimized using offline RL.

invoke (Serban et al., 2017a). Ziegler et al. (2019) used explicit human feedback to improve the summarization and text continuation performance of a large-scale language model.

Although implicit signals such as sentiment (Hancock et al., 2019) and conversation length (Zhou et al., 2018) have been used in maximum likelihood estimation (MLE) systems, the idea of using such signals as a reward for RL is relatively unexplored. Henderson et al. (2008) combine using reinforcement learning to optimize dialog reward with using supervised learning to restrict the conversation to be close to the training data. Shin et al. (2019) use on-policy learning in conjunction with a user-sentiment approximator to improve a seq 2 seq model, but are unable to learn directly from user feedback. To the best of our knowledge, we are the first to use offline RL to train dialog models on real human interactions.

\subsection{Offline RL and KL-Control}

The approach we propose is based on KL-control, a branch of stochastic optimal control (SOC) (Stengel, 1986) where the Kullback-Leibler (KL) divergence from some distribution is used to regularize an RL policy (Abdolmaleki et al., 2018; Kappen et al., 2012; Rawlik et al., 2012; Todorov, 2007). Well-known examples include Trust Region Policy Optimization (TRPO) (Schulman et al., 2015), and use conservative, KL-regularized policy updates to restrict the RL algorithm to stay close to its own prior policy (Haarnoja et al., 2018; Kakade, 2002; Peters et al., 2010; Rawlik et al., 2012). KL-control has been used to improve transfer learning between maximum likelihood estimation (MLE) training on 
data, and training with RL (Jaques et al., 2017). Our work is the first to propose KL-control from a pre-trained model to improve offline RL.

Other strategies to improve off-policy learning differ from our work: They either have focused on scenarios where the policy is able to explore and collect more data (Degris et al., 2012; Riedmiller, 2005) such as learning online from an outdated replay buffer (e.g. (Munos et al., 2016)), or have performed off-policy policy evaluation (Farajtabar et al., 2018; Jiang and Li, 2016; Precup, 2000; Thomas and Brunskill, 2016). In contrast, we learn a policy entirely offline, from a fixed batch of data, with no ability to explore. Others have tackled this problem using deep learning, but have not used KL-control (Liu et al., 2019; Gelada and Bellemare, 2019; Bhatt et al., 2019; Kumar et al., 2019; Agarwal et al., 2019; Fujimoto et al., 2018; Ghasemipour et al., 2020).

Most similar to our work is Batch Constrained Q-learning (BCQ) (Fujimoto et al., 2018), which addresses extrapolation error in offline RL by constraining the actions of the policy to be close to the offline data. This is accomplished by learning a generative model of the offline data, $p(a \mid s)$, and sampling from this model during learning and inference. We improve upon this approach by using KL-control to directly integrate knowledge of the prior model $p(a \mid s)$ into the RL policy.

\section{Way Off-Policy RL}

We adapt typical RL notation to the problem of generating a conversation. Here, we consider human interaction to represent the RL environment. The conversation history is the state $s_{t}$ of the environment at timestep $t$, and is composed of a series of utterances, which are composed of vocabulary tokens. The action $a_{t}$ that the RL model must take at each timestep is to select the most appropriate token according to its policy $\pi\left(a_{t} \mid s_{t}\right)$. Once it has constructed an utterance, the response of a human to that utterance is used to compute a reward signal $r_{t}$ to train the model. The agent's goal is to maximize reward over a conversation trajectory $\tau$, with a discount factor of $\gamma$ applied to future rewards.

$Q$-learning methods learn an action-value estimate of the total expected discounted future reward, $Q_{\pi}\left(a_{t}, s_{t}\right)=\mathbb{E}_{\pi}\left[\sum_{t^{\prime}=t}^{T} \gamma^{t^{\prime}-t} r_{t^{\prime}}\right]$, through iterative updates based on the Bellman equation:

$$
\begin{aligned}
& Q_{\theta_{\pi}}\left(s_{t}, a_{t}\right)=r_{t}+ \\
& \gamma \mathbb{E}_{s_{t+1} \sim p\left(\cdot \mid s_{t}, a_{t}\right)}\left[\max _{a_{t+1}} Q_{\theta_{T}}\left(s_{t+1}, a_{t+1}\right)\right]
\end{aligned}
$$

In deep $Q$-learning (Mnih et al., 2013), a $Q$ network approximates $Q_{\theta_{\pi}}\left(s_{t}, a_{t}\right)$ and drives the policy $\pi$. A second Target $Q$-network approximates the expected reward from the next state, $Q_{\theta_{T}}\left(s_{t+1}, a_{t+1}\right)$ (Van Hasselt et al., 2016). Here, we used pre-trained language models to initialize our $Q$ - and Target $Q$ - networks.

\subsection{Offline RL and extrapolation error}

In offline RL, we are given a fixed batch of data $\mathcal{B}$, and assume that no further interaction with the environment is possible. To train $Q_{\theta_{\pi}}$, we sample $\left(s_{t}, a_{t}, r_{t}, s_{t+1}\right) \sim \mathcal{B}$, and update the weights of the $Q$-network to approximate Eq. 1. Because $Q$-learning is an off-policy algorithm, in principle it should be able to learn from data collected by any behavior policy. However, extrapolation error occurs when the ORL policy learns to favor a state-action pair $(a, s)$ that is unlikely, or not contained, in the batch data. In this case, the estimate $Q(a, s)$ can be arbitrarily bad (Fujimoto et al., 2018). Because the Bellman equation bootstraps each $Q$-value based on all future $Q$ estimates, any error can accumulate to distort $Q$-values (Kumar et al., 2019). Experiments from Fujimoto et al. (2018) show that extrapolation error can be highly detrimental to offline RL.

These problems are compounded by the fact that algorithms like $Q$-learning are inherently optimistic in the face of uncertainty. When value estimates for some region of the state-action space are noisy (because too few experience samples have been used to refine them), the maximum operation in Eq. 1 will lead to an overestimation of expected reward. In a normal RL setting, this overestimation bias drives the model to explore states and actions for which the value estimates have the highest variance, thus enabling it to refine them; in essence, creating a built-in drive to explore. In the offline setting, where exploration is not possible, the model is instead driven to value parts of the state-action space for which it has little to no data to learn a good policy. Table 1 shows an example of this effect, where a vanilla $Q$-learning model trained on an offline batch of data (Batch $Q$ ) begins to use unrealistic language that is not contained within the 
[User]: hey, what's up?

[Batch Q]: where did you get??, that sounds so exciting. how do you work for you... you want to talk to me?... you want [User]: yes, i want to talk to you!

[Batch Q]: where did you say to me?... you want to talk to you about you... you are so good for you... you are so good...
[User]: hey, what's up?

[KL-control]: hey, i hope you have a great day and i wish you the best.

[User]: oh thank you that's very sweet of you.

[KL-control]: so, i'm so excited, and i'm so excited to meet new people.

Table 1: Purely reward-maximizing methods like Batch $Q$ trivially exploit a reward for asking questions by only asking questions, and using the maximum number of tokens in every sentence. In contrast, KL-control methods output plausible language by staying close to the language prior, while eliciting positive feedback from humans.

batch data, for example saying implausible phrases such as "where did you say to me?".

Even in the online setting, applying deep RL to dialog generation is challenging due to the large state-action space. While typical game RL tasks may have an action space of dimension 8 (Mnih et al., 2013), in dialog the action space is the number of tokens in the vocabulary: 20,000. The highdimensional state-action space further compounds the problems of extrapolation error and overestimation bias in offline RL. Below, we describe a novel method to ameliorate these issues.

\subsection{Dropout for uncertainty estimation of Target $Q$-values}

Overestimation error in estimating future rewards based on Target $Q$-values poses an issue for offline RL. We leverage the fact that a network trained with dropout can be used to approximate a Bayesian uncertainty estimate of the network's output (Gal and Ghahramani, 2016). Given the target $Q$-network $Q_{\theta_{T}}$, we compute $Q\left(a_{t+1}, s_{t+1}\right)$ by running $M$ stochastic forward passes of the network, each with a new dropout mask $d_{i}$. Taking the minimum of these outputs gives a Monte Carlo (MC) estimate of the lower-bound of $Q_{\theta_{T}}\left(a_{t+1}, s_{t+1}\right)$ :

$$
Q\left(a_{t+1}, s_{t+1}\right)=\min _{i=1 \ldots M}\left[Q_{\theta_{T}}\left(a_{t+1}, s_{t+1} ; d_{i}\right)\right]
$$

This penalizes high variance estimates and leads the algorithm to be pessimistic in the face of uncertainty, rather than optimistic, favoring actions and states well covered by the offline data.

\subsection{KL Control from pre-trained prior}

Recall that BCQ (Fujimoto et al., 2018) uses offline data to learn a model of which actions are probable given a state: $p(a \mid s)$. It then samples actions from $p(a \mid s)$ to constrain the RL policy such that it cannot take unrealistic actions.

In the language domain, we already have access to a better model of $p(a \mid s)$ than could easily be learned from a small amount of offline data. Any language model gives us the probability of a word occurring given a particular conversation context $(p(a \mid s))$, and can be used as a language prior to prevent the RL model from choosing unrealistic words. Rather than simply sampling from this prior, we directly incorporate knowledge of the prior into the RL policy. To achieve this, we use KL-control to penalize divergence between the prior $p(a \mid s)$ and the $Q$-network policy $\pi_{\theta}$, while maximizing reward.

Given a trajectory of actions, $\tau=$ $\left\{a_{1}, a_{2}, \ldots a_{t-1}\right\}$, let $q(\tau)=\prod_{t=1}^{T} \pi_{\theta}\left(a_{t}, s_{t}\right)$ be the policy of our $Q$-learning algorithm at the trajectory level. Similarly, let $p(\tau)=\prod_{t=1}^{T} p\left(a_{t} \mid s_{t}\right)$ be the prior distribution over the trajectory, and $r(\tau)$ be the rewards. We seek to maximize the following KL-regularized objective:

$$
L(q)=\mathbb{E}_{q(\tau)}[r(\tau)] / c-D_{K L}[q(\tau) \| p(\tau)]
$$

As $D_{K L}[q \| p]=\sum_{x} q(x)(\log q(x)-\log p(x))$, this is equivalent to maximizing the following expected value function at the action level:

$$
\begin{aligned}
Q^{\pi}\left(s_{t}, a_{t}\right) & =\mathbb{E}_{\pi}\left[\sum_{t^{\prime}=t}^{T} r\left(s_{t^{\prime}}, a_{t^{\prime}}\right) / c\right. \\
& \left.+\log p\left(a_{t^{\prime}} \mid s_{t^{\prime}}\right)-\log \pi\left(a_{t^{\prime}} \mid s_{t^{\prime}}\right)\right]
\end{aligned}
$$

The two terms we have introduced in Eq. 3 have clear implications. The $\log p(a \mid s)$ term rewards choosing actions that have high probability under the prior, biasing the model to state-action pairs that are realistic and likely to be in the offline data; thus, extrapolation error is reduced. The effects of using KL-control to ensure an RL model continues to use realistic language are shown in Table 1.

The $-\log \pi(a \mid s)$ term is analogous to entropy regularization. Maintaining diversity through entropy regularization is important for dialog models, which are known to collapse to a small number of uninteresting samples (Li et al., 2017a). 
We can derive an entropy-regularized version of $Q$-learning, known as soft $Q$-learning (Haarnoja et al., 2017), or $\Psi$-learning (Jaques et al., 2017; Rawlik et al., 2012). This allows us to re-state our entropy-regularized, KL-control objective as:

$$
\begin{aligned}
\Psi^{*}\left(s_{t}, a_{t}\right) & =r\left(s_{t^{\prime}}, a_{t^{\prime}}\right) / c+\log p\left(a_{t^{\prime}} \mid s_{t^{\prime}}\right) \\
& +\gamma \log \sum_{a^{\prime}} \exp \left(\Psi^{*}\left(s^{\prime}, a^{\prime}\right)\right) \\
\pi_{\Psi}^{*}\left(a_{t} \mid s_{t}\right) & =\exp \left(\Psi^{*}\left(s_{t}, a_{t}\right)\right)
\end{aligned}
$$

Because it avoids taking a hard max over noisy estimates, this $\Psi$-learning objective leads to less overestimation of future reward, and aids learning through more stable temporal-difference updates.

\subsection{Comparison to existing techniques}

To test our algorithm against a state-of-the-art offline deep RL technique, we implement a discrete version of Batch Constrained Q-learning (Fujimoto et al., 2018), DBCQ. For a fair comparison, we also use a fully trained language model to provide $p(a \mid s)$ to BCQ, and apply our Monte Carlo target estimation technique to reduce overestimation error. Finally, to adapt BCQ to discrete action spaces, we remove the continuous-action perturbation model.

\section{Learning from talking to humans}

Figure 1 illustrates our experimental approach. The left side of the figure describes traditional approaches to dialog generation, in which human feedback is only used to evaluate static conversations generated by dialog models. In contrast, we allow humans to freely interact with our models online, and use their implicit conversation cues to update our dialog models using offline RL.

\subsection{Training baseline dialog models}

Before learning from human feedback with RL, we first train a collection of baseline dialog models using standard corpora: the CORNELL dataset of movie dialog (Danescu-Niculescu-Mizil and Lee, 2011) and a REDDIT Casual Conversations dataset (Ghandeharioun et al., 2019). For model architectures, we focused on hierarchical sequence-tosequence models (Serban et al., 2016, 2017b; Park et al., 2018)

because they were found to be more effective for the datasets under consideration than e.g. Transformers (Saleh et al., 2019). Regardless, the techniques proposed here are model-agnostic, and could be applied to a dialog model with any underlying architecture. In total, we trained over 40 dia$\log$ models with different architectures, on different datasets, with different feature-based regularization (e.g. sentiment or relatedness as in Ghandeharioun et al. (2019)). These models vary significantly in the distribution of language they learned, and thus differ significantly from the offline RL policy.

\subsection{Hosting real-time conversations online}

The trained models were deployed to interact live with human users via a web server that hosts neural network dialog models on GPU for fast, real-time inference: https: //github.com/asmadotgh/neural_chat_web.

Figure 2 shows a screenshot of the interface, which includes buttons that allow users to give manual feedback on responses they particularly liked or disliked. Users were encouraged to use these buttons, and we sum these manual votes to create an overall votes score. After chatting, users were asked to provide a Likert scale rating of the bot's conversation quality, fluency, diversity, contingency/relatedness, and empathy. The code for the RL models is available in open-source at https://github.com/natashamjaques/ neural_chat/tree/master/BatchRL. Using the server, we collected a batch of human interaction data containing 46,061 pairs of user input and agent response. Because humans may use inappropriate language with bots online (see (Horton, 2016)), we filtered this data to remove 1 character responses, profanities, and invalid inputs for a remaining total of 45,179 response pairs. This filtering step is important to ensure undesirable human behavior is not learned by the $\mathrm{RL}$ algorithms. The offline data was used to train the RL models as described in Section 3.

\subsection{Evaluating offline RL models}

We recruited 80 Mechanical Turk workers to provide a total of 600 7-point Likert scale ratings of the trained bots, after interacting with each for at least 6 turns. We note that using this platform to test our models "in the wild" with novel humans represents a more meaningful test of generalization than testing an RL model in the same limited (game) environment in which it was trained, since humans are not restricted in the text they can type as input to the model. 


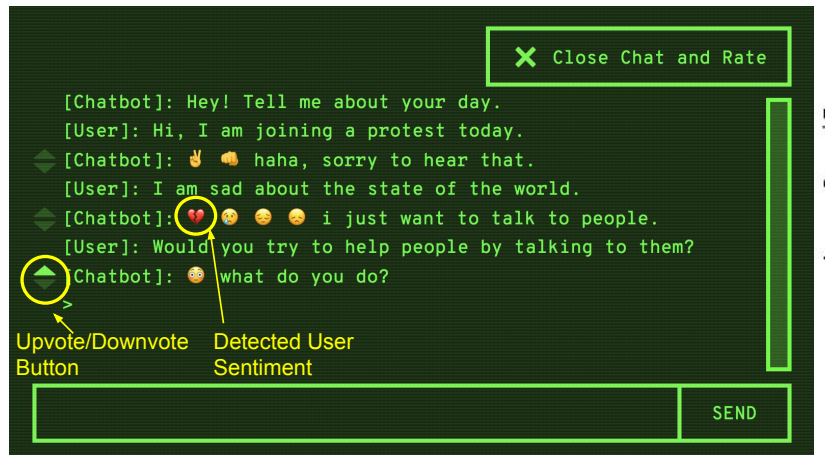

(a) Platform Interface

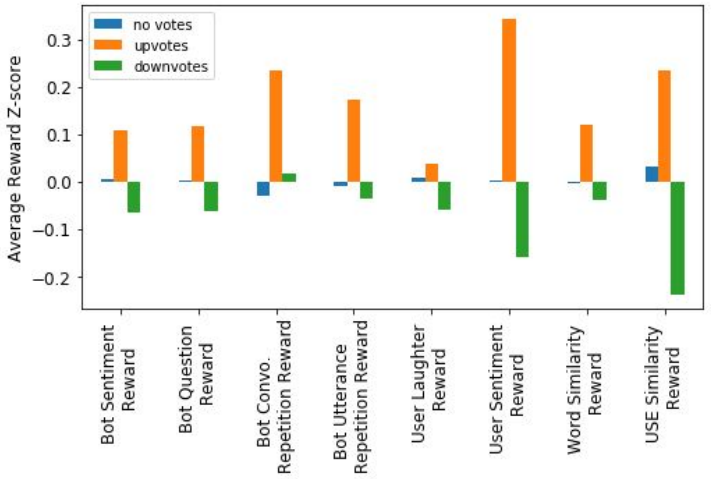

(b) Rewards by Upvote/Downvote

Figure 2: (a) Platform interface in which users chat in real time with dialog models hosted on GPU. The interface displays the user's sentiment detected with DeepMoji (Felbo et al., 2017), and includes buttons for the user to upvote (downvote) a response they particularly like (dislike). (b) By conditioning on responses which received positive, neutral, and negative manual feedback (votes), we can determine which implicit rewards map most clearly to user ratings.

\section{Measuring implicit conversation cues}

Our goal is to improve a dialog model's ability to engage in natural conversation with a human by learning from the implicit signals in the human's response. Requiring a human to manually rate good interactions is unnatural and cumbersome, and we hypothesize it cannot scale as effectively as recognizing and learning from informative cues within the user's text responses. The golden question is which goals should be used to train a good chit-chat dialog model.

Understanding when a human is satisfied with the conversation is an unsolved problem. As a first step, we designed several intrinsic conversation rewards, taking inspiration from prior work in dialog, as well as the psychology of human conversation. We noted that psychologists have identified the importance of emotion in creating a sense of understanding (Bodie et al., 2015; Weger Jr et al., 2010), laughter as important to building solidarity (Hay, 2000), paraphrasing and style matching as helping to facilitate good conversation (Ireland et al., 2011; Weger Jr et al., 2010), and asking questions as an important active listening skill (Bodie et al., 2012). Further, prior work has found that eliciting longer conversations can be a signal of engagement (Sidner et al., 2004; Zhou et al., 2018), and that reducing repetition and increasing specificity on the part of the model can improve conversation quality (See et al., 2019; Mehri and Eskenazi, 2020). We compute a large collection (30 in total) of bot rewards (rewards based on bot behavior e.g. asking questions), user rewards (rewards based on eliciting positive user behavior e.g. laughter), and interac- tion rewards (rewards based on similarity between the user's input and bot's response e.g. similarity to the user's response in sentence embedding space).

To determine which of these rewards objectively relate to user satisfaction, we examine the reward score for those responses that received positive, negative, and neutral manual feedback using the upvote/downvote buttons provided in the interface. We found that only some of the rewards mapped accurately to user ratings (see Figure 2b), and these are the ones we optimize with our RL models. For more details about the reward functions, please see the appendix. Notably, conversation length and specificity score were not found to be higher in upvoted bot responses.

Note that four of the rewards (starting with the bot prefix) can be optimized by the model itself, but the remaining four rewards include eliciting positive responses from a human user or measuring user-bot response similarity (e.g. using word overlap or similarity in Universal Sentence Encoder (USE) embeddings (Cer et al., 2018)).

\section{Results}

\subsection{Controlling bot conversation behavior}

We first examine whether our algorithms can successfully maximize the proposed bot rewards as intended ${ }^{1}$. We trained RL models on 1) bot sentiment reward only, 2) user sentiment reward only, and 3) a combination of rewards (from Figure 2b). We compare the effectiveness of these models to a

\footnotetext{
${ }^{1}$ In the appendix, we provide a study comparing WOP to prior work in traditional, non-dialog RL tasks, and find that it outperforms all relevant baselines including DBCQ.
} 


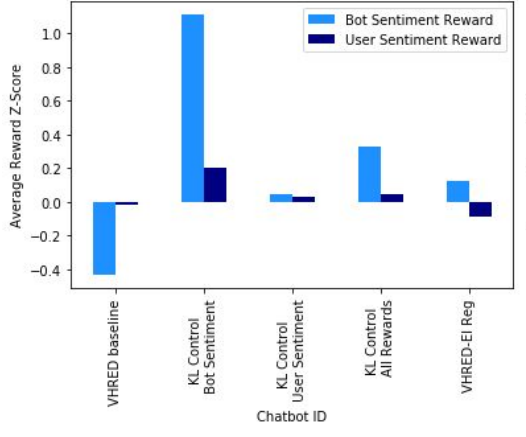

(a) Sentiment Rewards

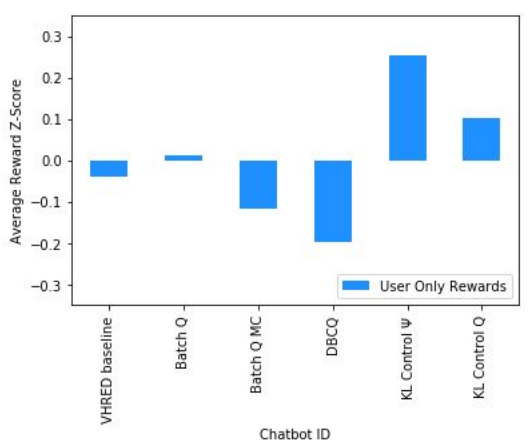

(b) User Rewards

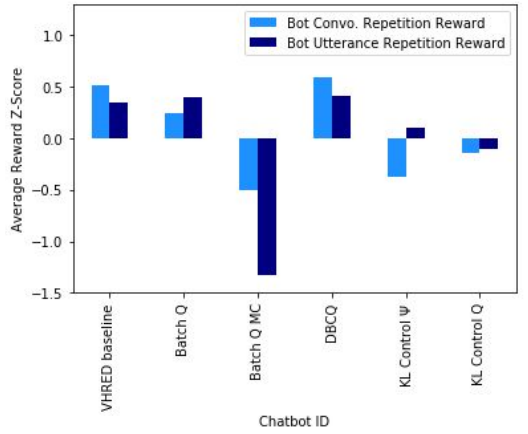

(c) Bot Repetition Rewards

Figure 3: (a) Average reward scores of sentiment rewards computed on study chat transcripts across different models. KLcontrol methods more effectively increase bot sentiment and elicit more positive sentiment from humans than either the baseline language model or adding sentiment regularizer during supervised training. (b) The sentiment and laughter elicited from humans is higher for KL-control methods than the language model baseline and other offline RL techniques. (c) Average bot repetition reward scores (higher scores indicate less repetition). The RL models contain more conversation and utterance repetition.

baseline VHRED model and a Sentiment and Infersent regularized VHRED model (as proposed by Ghandeharioun et al. (2019)). We compute the reward scores (e.g. sentiment) based on conversations with new humans in the wild (i.e. during the final study). Figure 3a shows that the KL-control model, trained to maximize bot sentiment, achieves higher bot sentiment in experiments than both the VHRED baseline and the VHRED-EI model (with sentiment and topic regularization (Ghandeharioun et al., 2019)). This illustrates that for controlling bot sentiment, a reward-based approach better optimizes bot behavior than training with sentimentbased regularization. Furthermore, controlling bot sentiment also leads to eliciting higher user sentiment in our open-domain experiments.

\subsection{Measuring human conversation behavior}

We then consider how effective our algorithms are at maximizing rewards that are based on human behavior.

Although user rewards are inherently more difficult to optimize than bot rewards, Figure $3 b$ illustrates that our KL-control models elicit higher human reward scores (user sentiment and user laughter) than other offline RL algorithms and the baseline VHRED model. This demonstrates the success of our algorithms in eliciting positive responses from the human conversation participants ${ }^{2}$.

\subsection{Overall human ratings}

Table 2 shows the results of the human evaluation, comparing WOP to ablations of itself, vanilla of-

\footnotetext{
${ }^{2}$ In the appendix, we replicate these experiments with a different baseline model, and produce the same findings.
}

fline RL (Batch $Q$ ), and DBCQ.

Compared to the RL baseline (Batch $Q$ ), MC Target $Q$ estimation leads to modest improvements in Fluency. While the DBCQ model is rated better than Batch $Q$ and does well in the Diversity category, it performs worse than the WOP KL-control methods, particularly at eliciting human rewards. The KL-control models show substantial gains over the RL baselines across both ratings and human reward. We perform a one-way analysis of variance (ANOVA) comparing the KL-control models to the Batch $Q$ baselines and DBCQ on total human ratings, and find that the KL-control models are significantly better, $F(x)=7.328, p<.005$. This validates the hypothesis that KL-control with a strong, pre-trained prior can be used to improve offline RL.

\subsection{The role of repetition}

The overall human quality ratings are worse in the offline RL bots as compared to the language model prior (Table 2). The biggest gap between the VHRED and RL models is the diversity ratings. The conversation and utterance repetition scores of each technique in Figure 3c reveal that the RL models (including the KL-control models) contain more repetition than the baseline. We hypothesize that due to the limited size of our offline data, the RL models have restricted their outputs to focus on a narrow range of conversations that elicited high rewards in the training data, which may increase repetitiveness. Some applications may require shaping dialog model behavior towards a desired objective (such as using appropriate language) over maximizing other conversation objectives. 


\begin{tabular}{|l|lllll|l|r|r|}
\hline Model type & Quality & Fluency & Diversity & Relatedness & Empathy & Total & Votes & $\begin{array}{l}\text { Human } \\
\text { reward }\end{array}$ \\
\hline VHRED-Baseline & $2.65 \pm .46$ & $3.83 \pm .47$ & $4.05 \pm .52$ & $2.43 \pm .44$ & $3.08 \pm .53$ & $16.03 \pm 1.93$ & 0.27 & -0.04 \\
\hline DBCQ & $1.80 \pm .41$ & $1.49 \pm .29$ & $\mathbf{3 . 2 2} \pm .57$ & $1.56 \pm .25$ & $2.10 \pm .37$ & $10.17 \pm 1.29$ & -0.07 & -0.20 \\
Batch $Q$ & $1.30 \pm .19$ & $2.85 \pm .54$ & $1.15 \pm .13$ & $1.23 \pm .15$ & $2.18 \pm .55$ & $8.70 \pm 0.97$ & -0.16 & 0.01 \\
Batch $Q+$ MC & $1.53 \pm .24$ & $2.15 \pm .37$ & $1.60 \pm .32$ & $1.53 \pm .28$ & $\mathbf{2 . 5 8} \pm .48$ & $9.38 \pm 1.31$ & -0.21 & -0.12 \\
KL-control $Q$ & $\mathbf{2 . 2 3} \pm .44$ & $\mathbf{2 . 8 8} \pm .41$ & $2.65 \pm .41$ & $\mathbf{2 . 1 5} \pm .39$ & $2.28 \pm .47$ & $\mathbf{1 2 . 1 8} \pm \mathbf{1 . 5 9}$ & $\mathbf{0 . 0 9}$ & 0.10 \\
KL-control $\Psi$ & $1.98 \pm .44$ & $2.73 \pm .45$ & $2.30 \pm .42$ & $1.90 \pm .37$ & $2.40 \pm .44$ & $11.30 \pm 1.63$ & 0.04 & $\mathbf{0 . 2 5}$ \\
\hline
\end{tabular}

Table 2: Interactive human evaluation of offline RL techniques (best RL model bolded). KL-control strongly outperforms other offline RL techniques. Ratings are Likert scale with 95\% confidence intervals $(n=40)$. Votes and human reward are $z$-scores.

\begin{tabular}{|l|lllll|l|r|r|}
\hline $\begin{array}{l}\text { Reward } \\
\text { function }\end{array}$ & Quality & Fluency & Diversity & Relatedness & Empathy & Total & Votes & $\begin{array}{l}\text { Human } \\
\text { reward }\end{array}$ \\
\hline Manual votes & $2.53 \pm .51$ & $3.43 \pm .52$ & $2.88 \pm .50$ & $2.40 \pm .45$ & $3.30 \pm .45$ & $14.53 \pm 1.96$ & -0.05 & -0.07 \\
User laughter & $2.53 \pm .47$ & $3.38 \pm .50$ & $\mathbf{3 . 0 5} \pm .47$ & $2.25 \pm .43$ & $3.08 \pm .48$ & $14.28 \pm 1.96$ & 0.06 & 0.01 \\
User Sentiment & $\mathbf{2 . 6 0} \pm .49$ & $3.30 \pm .50$ & $2.90 \pm .50$ & $2.38 \pm .47$ & $3.23 \pm .55$ & $14.40 \pm 2.25$ & 0.04 & 0.05 \\
Word Similarity & $2.58 \pm .52$ & $3.53 \pm .49$ & $2.98 \pm .50$ & $2.45 \pm .45$ & $3.08 \pm .46$ & $14.60 \pm 2.00$ & 0.02 & -0.18 \\
USE Similarity & $2.05 \pm .41$ & $3.65 \pm .48$ & $2.38 \pm .46$ & $2.03 \pm .45$ & $2.75 \pm .46$ & $12.85 \pm 1.77$ & -0.11 & -0.11 \\
Bot Question & $2.43 \pm .52$ & $3.65 \pm .52$ & $2.63 \pm .47$ & $2.65 \pm .51$ & $2.70 \pm .48$ & $14.05 \pm 2.14$ & 0.01 & 0.09 \\
Bot Sentiment & $1.90 \pm .45$ & $3.20 \pm .53$ & $1.88 \pm .52$ & $1.88 \pm .46$ & $3.20 \pm .41$ & $12.05 \pm 1.91$ & -0.04 & $\mathbf{0 . 1 4}$ \\
Bot Repetition & $2.48 \pm .45$ & $\mathbf{3 . 7 8} \pm . \mathbf{4 9}$ & $2.95 \pm .52$ & $\mathbf{2 . 6 3} \pm .45$ & $\mathbf{3 . 6 5} \pm . \mathbf{6 1}$ & $\mathbf{1 5 . 4 8} \pm \mathbf{1 . 9 7}$ & $\mathbf{0 . 0 7}$ & 0.05 \\
\hline
\end{tabular}

Table 3: Interactive human evaluation of WOP trained with different reward functions. Manual votes are outperformed by implicit signals. Ratings are Likert scale with 95\% confidence intervals $(n=40)$, votes and human reward are $z$-scores.

\subsection{Comparing rewards}

Table 3 presents the results of models trained with only a single reward function, to investigate which rewards presented in Section 5 are useful for achieving high-quality conversations with humans.

We note that extracting a set of reward functions post-hoc from a batch of data and training on these independently is made feasible through offline RL. Here all models are trained with WOP (KL-control, $\Psi$-learning, and MC targets). Maximizing positive sentiment in the user leads to the highest quality bot, underscoring the importance of implicit signals as cues for good conversation. The bot trained on the manual votes provided by users at the utterance level achieves decent quality scores, but fails to elicit a higher z-score of manual upvotes than other models.

Training on the manual upvote reward may help the bot learn successful behaviors indirectly but such a sparse reward is difficult to optimize for directly. Even though users were instructed to make use of the vote feature, voting is burdensome, and users did not vote frequently enough to provide a good training signal.

Meanwhile, implicit signals of human enjoyment (such as sentiment) are dense and thus a more scalable way to learn from human preferences. Across all bots trained on single features, the bot trained on minimizing repetition (both on a conversational and utterance level) achieves the best quality over all.

\section{Discussion}

In this work, we present novel techniques that enable successful offline reinforcement learning on any base language model from real human conversations. This allows the dialog systems practitioner to train models that learn language structure from vast, readily-available corpora, then fine-tune for specific desirable behaviors post-hoc through RL rewards.

We observe that the new offline RL method successfully optimizes both generated bot rewards and elicited human responses. We show that it presents a better option than using regularization in training a specific bot behavior. Further, RL currently remains the only option for maximizing user feedback over the course of a conversation.

Compared to prior work in offline RL, the novel WOP offline RL algorithm achieves higher performance in traditional RL tasks, elicits more positive feedback in conversations with novel humans at test time, and earns overall higher human ratings.

A limitation of our study is that the question of what to optimize with RL to improve overall qualitative ratings remains open. We have shown that manual ratings are too sparse to optimize effectively, and instead suggest using implicit rewards. 
However, our reward set proved insufficient to achieve higher human quality ratings, at least with the limited offline training data we were able to collect. It is unlikely the rewards proposed here fully cover what it means to have a high quality openended conversation. Future work should investigate more rewards for training an open-domain dialog model such as long term conversation rewards that may need to be computed over many conversation turns.

Our work computes conversational rewards based on dialog data and annotations from online task workers in the United States. Considering the broader impacts of our work, a representative and diverse set of conversations and annotations should be collected before real world systems are trained and deployed using our algorithms.

We have shown that the proposed techniques can be useful for shaping dialog model behavior towards a desired objective. For many practical applications, we may have specific requirements for the language generated by a model-for example, that it is appropriate, positive, and polite-even if this leads to a lower perception of conversation quality for some users. We have shown that the Way Off-Policy algorithm provides a more effective way to teach a language model specific behaviors from offline data than previously proposed RL or regularization techniques.

\section{Acknowledgments}

We would like to thank Scott Fujimoto for insightful email correspondence on this topic, approval of the DBCQ algorithm, and suggestion to apply model averaging. We would like to thank Sudha Rao and Yonatan Bisk for helpful guidance and feedback in the re-framing and re-writting process of this work. We also thank Max KleimanWeiner, Ardavan Saeedi, Sebastian Zepf, Sara Taylor, Oliver Saunders Wilder, Kyle Kastner, Marissa Zhang, and Kristy Johnson for their helpful discussions about this project, and many others for helping test-drive our bots.

We thank the MIT Quest for Intelligence, and MIT Stephen A. Schwarzman College of Computing, and the Machine Learning Across Disciplines Challenge for providing computing resources, and MIT Media Lab Consortium for the support of this research. This work has been partially supported by RTI2018-095232-B-C22 grant from the Spanish Ministry of Science.

\section{References}

Abbas Abdolmaleki, Jost Tobias Springenberg, Yuval Tassa, Remi Munos, Nicolas Heess, and Martin Riedmiller. 2018. Maximum a posteriori policy optimisation. International Conference on Learning Representations.

Daniel Adiwardana, Minh-Thang Luong, David R So, Jamie Hall, Noah Fiedel, Romal Thoppilan, Zi Yang, Apoorv Kulshreshtha, Gaurav Nemade, Yifeng Lu, et al. 2020. Towards a human-like open-domain chatbot. arXiv preprint arXiv:2001.09977.

Rishabh Agarwal, Dale Schuurmans, and Mohammad Norouzi. 2019. Striving for simplicity in offpolicy deep reinforcement learning. arXiv preprint arXiv:1907.04543.

Aditya Bhatt, Max Argus, Artemij Amiranashvili, and Thomas Brox. 2019. Crossnorm: Normalization for off-policy td reinforcement learning. arXiv preprint arXiv:1902.05605.

Graham D Bodie, Kellie St. Cyr, Michelle Pence, Michael Rold, and James Honeycutt. 2012. Listening competence in initial interactions i: Distinguishing between what listening is and what listeners do. International Journal of Listening, 26(1):1-28.

Graham D Bodie, Andrea J Vickery, Kaitlin Cannava, and Susanne M Jones. 2015. The role of "active listening" in informal helping conversations: Impact on perceptions of listener helpfulness, sensitivity, and supportiveness and discloser emotional improvement. Western Journal of Communication, 79(2):151-173.

Greg Brockman, Vicki Cheung, Ludwig Pettersson, Jonas Schneider, John Schulman, Jie Tang, and Wojciech Zaremba. 2016. Openai gym.

Daniel Cer, Yinfei Yang, Sheng-yi Kong, Nan Hua, Nicole Limtiaco, Rhomni St John, Noah Constant, Mario Guajardo-Cespedes, Steve Yuan, Chris Tar, et al. 2018. Universal sentence encoder. arXiv preprint arXiv:1803.11175.

Alexis Conneau, Douwe Kiela, Holger Schwenk, Loïc Barrault, and Antoine Bordes. 2017. Supervised learning of universal sentence representations from natural language inference data. In Proceedings of the 2017 Conference on Empirical Methods in Natural Language Processing, pages 670-680.

Cristian Danescu-Niculescu-Mizil and Lillian Lee. 2011. Chameleons in imagined conversations: A new approach to understanding coordination of linguistic style in dialogs. In Proceedings of the 2nd Workshop on Cognitive Modeling and Computational Linguistics, pages 76-87. Association for Computational Linguistics.

Thomas Degris, Martha White, and Richard S Sutton. 2012. Off-policy actor-critic. In Proceedings of the 
29th International Coference on International Conference on Machine Learning, pages 179-186. Omnipress.

Mehrdad Farajtabar, Yinlam Chow, and Mohammad Ghavamzadeh. 2018. More robust doubly robust offpolicy evaluation. In International Conference on Machine Learning, pages 1446-1455.

Mehdi Fatemi, Layla El Asri, Hannes Schulz, Jing He, and Kaheer Suleman. 2016. Policy networks with two-stage training for dialogue systems. In Proceedings of the 17th Annual Meeting of the Special Interest Group on Discourse and Dialogue, pages 101110.

Bjarke Felbo, Alan Mislove, Anders Søgaard, Iyad Rahwan, and Sune Lehmann. 2017. Using millions of emoji occurrences to learn any-domain representations for detecting sentiment, emotion and sarcasm. In 2017 Conference on Empirical Methods in Natural Language Processing Conference on Empirical Methods in Natural Language Processing. Association for Computational Linguistics.

Scott Fujimoto, David Meger, and Doina Precup. 2018. Off-policy deep reinforcement learning without exploration. arXiv preprint arXiv:1812.02900.

Yarin Gal and Zoubin Ghahramani. 2016. Dropout as a bayesian approximation: Representing model uncertainty in deep learning. In international conference on machine learning, pages 1050-1059.

Milica Gašić, Filip Jurčíček, Blaise Thomson, Kai Yu, and Steve Young. 2011. On-line policy optimisation of spoken dialogue systems via live interaction with human subjects. In 2011 IEEE Workshop on Automatic Speech Recognition \& Understanding, pages 312-317. IEEE.

Carles Gelada and Marc G Bellemare. 2019. Offpolicy deep reinforcement learning by bootstrapping the covariate shift. arXiv preprint arXiv:1901.09455.

Asma Ghandeharioun, Judy Hanwen Shen, Natasha Jaques, Craig Ferguson, Noah Jones, Agata Lapedriza, and Rosalind Picard. 2019. Approximating interactive human evaluation with self-play for open-domain dialog systems. In Advances in $\mathrm{Neu}$ ral Information Processing Systems, pages 1365813669.

Seyed Kamyar Seyed Ghasemipour, Dale Schuurmans, and Shixiang Shane Gu. 2020. Emaq: Expected-max q-learning operator for simple yet effective offline and online rl. arXiv preprint arXiv:2007.11091.

Herbert P Grice. 1975. Logic and conversation. In Speech acts, pages 41-58. Brill.

Tuomas Haarnoja, Haoran Tang, Pieter Abbeel, and Sergey Levine. 2017. Reinforcement learning with deep energy-based policies. In Proceedings of the 34th International Conference on Machine Learning-Volume 70, pages 1352-1361. JMLR. org.

Tuomas Haarnoja, Aurick Zhou, Pieter Abbeel, and Sergey Levine. 2018. Soft actor-critic: Off-policy maximum entropy deep reinforcement learning with a stochastic actor. In International Conference on Machine Learning, pages 1856-1865.

Braden Hancock, Antoine Bordes, Pierre-Emmanuel Mazare, and Jason Weston. 2019. Learning from dialogue after deployment: Feed yourself, chatbot! arXiv preprint arXiv:1901.05415.

Jennifer Hay. 2000. Functions of humor in the conversations of men and women. Journal of pragmatics, 32(6):709-742.

James Henderson, Oliver Lemon, and Kallirroi Georgila. 2008. Hybrid reinforcement/supervised learning of dialogue policies from fixed data sets. Computational Linguistics, 34(4):487-511.

Geoffrey Hinton, Oriol Vinyals, and Jeff Dean. 2015 Distilling the knowledge in a neural network. arXiv preprint arXiv:1503.02531.

Helena Horton. 2016. Microsoft deletes 'teen girl' ai after it became a hitler-loving sex robot within 24 hours. In Telegraph UK.

Molly E Ireland, Richard B Slatcher, Paul W Eastwick, Lauren E Scissors, Eli J Finkel, and James W Pennebaker. 2011. Language style matching predicts relationship initiation and stability. Psychological science, 22(1):39-44.

Natasha Jaques, Shixiang Gu, Dzmitry Bahdanau, José Miguel Hernández-Lobato, Richard E Turner, and Douglas Eck. 2017. Sequence tutor: Conservative fine-tuning of sequence generation models with kl-control. In Proceedings of the 34th International Conference on Machine Learning-Volume 70, pages 1645-1654. JMLR. org.

Nan Jiang and Lihong Li. 2016. Doubly robust offpolicy value evaluation for reinforcement learning. In International Conference on Machine Learning, pages 652-661.

Sham M Kakade. 2002. A natural policy gradient. In Advances in neural information processing systems (NIPS), volume 14, pages 1531-1538.

Hilbert J Kappen, Vicenç Gómez, and Manfred Opper. 2012. Optimal control as a graphical model inference problem. Machine learning, 87(2):159-182.

Diederik P Kingma and Jimmy Ba. 2014. Adam: A method for stochastic optimization. arXiv preprint arXiv:1412.6980.

Aviral Kumar, Justin Fu, George Tucker, and Sergey Levine. 2019. Stabilizing off-policy q-learning via bootstrapping error reduction. arXiv preprint arXiv:1906.00949. 
Jiwei Li, Alexander H Miller, Sumit Chopra, Marc'Aurelio Ranzato, and Jason Weston. 2017a. Dialogue learning with human-in-the-loop. International Conference on Learning Representations.

Jiwei Li, Will Monroe, Alan Ritter, Dan Jurafsky, Michel Galley, and Jianfeng Gao. 2016. Deep reinforcement learning for dialogue generation. In Proceedings of the 2016 Conference on Empirical Methods in Natural Language Processing, pages 11921202.

Jiwei Li, Will Monroe, Tianlin Shi, Sébastien Jean, Alan Ritter, and Dan Jurafsky. 2017b. Adversarial learning for neural dialogue generation. In Proceedings of the 2017 Conference on Empirical Methods in Natural Language Processing, pages 2157-2169.

Ziming Li, Julia Kiseleva, and Maarten de Rijke. 2018. Dialogue generation: From imitation learning to inverse reinforcement learning. arXiv preprint arXiv:1812.03509.

Bing Liu and Ian Lane. 2017. Iterative policy learning in end-to-end trainable task-oriented neural dialog models. In 2017 IEEE Automatic Speech Recognition and Understanding Workshop (ASRU), pages 482-489. IEEE.

Bing Liu, Gokhan Tür, Dilek Hakkani-Tür, Pararth Shah, and Larry Heck. 2018. Dialogue learning with human teaching and feedback in end-to-end trainable task-oriented dialogue systems. In Proceedings of the 2018 Conference of the North American Chapter of the Association for Computational Linguistics. Human Language Technologies, Volume 1 (Long Papers), pages 2060-2069.

Yao Liu, Adith Swaminathan, Alekh Agarwal, and Emma Brunskill. 2019. Off-policy policy gradient with state distribution correction. ICML 2019 Workshop RLARealLife.

Shikib Mehri and Maxine Eskenazi. 2020. Unsupervised evaluation of interactive dialog with dialogpt. Proceedings of the SIGdial 2020 Conference.

Volodymyr Mnih, Koray Kavukcuoglu, David Silver, Alex Graves, Ioannis Antonoglou, Daan Wierstra, and Martin Riedmiller. 2013. Playing atari with deep reinforcement learning. NIPS Deep Learning Workshop.

Rémi Munos, Tom Stepleton, Anna Harutyunyan, and Marc Bellemare. 2016. Safe and efficient off-policy reinforcement learning. In Advances in Neural Information Processing Systems, pages 1054-1062.

Yookoon Park, Jaemin Cho, and Gunhee Kim. 2018. A hierarchical latent structure for variational conversation modeling. In Proceedings of the 2018 Conference of the North American Chapter of the Association for Computational Linguistics: Human Language Technologies, Volume 1 (Long Papers), pages 1792-1801.
Jan Peters, Katharina Mülling, and Yasemin Altun. 2010. Relative entropy policy search. In $A A A I$, pages 1607-1612. Atlanta.

Doina Precup. 2000. Eligibility traces for off-policy policy evaluation. Computer Science Department Faculty Publication Series, page 80.

Robert R Provine. 1996. Laughter. American scientist, 84(1):38-48

Konrad Rawlik, Marc Toussaint, and Sethu Vijayakumar. 2012. On stochastic optimal control and reinforcement learning by approximate inference. In Robotics: science and systems.

Martin Riedmiller. 2005. Neural fitted q iteration-first experiences with a data efficient neural reinforcement learning method. In European Conference on Machine Learning, pages 317-328. Springer.

Abdelrhman Saleh, Natasha Jaques, Asma Ghandeharioun, Judy Hanwen Shen, and Rosalind Picard. 2019. Hierarchical reinforcement learning for opendomain dialog. The Thirty-Fourth AAAI Conference on Artificial Intelligence.

John Schulman, Sergey Levine, Pieter Abbeel, Michael Jordan, and Philipp Moritz. 2015. Trust region policy optimization. In Proceedings of the 32nd International Conference on Machine Learning (ICML15), pages 1889-1897.

Abigail See, Stephen Roller, Douwe Kiela, and Jason Weston. 2019. What makes a good conversation? how controllable attributes affect human judgments. North American Chapter of the Association for Computational Linguistics: Human Language Technologies (NAACL-HLT 2019).

Iulian V Serban, Chinnadhurai Sankar, Mathieu Germain, Saizheng Zhang, Zhouhan Lin, Sandeep Subramanian, Taesup Kim, Michael Pieper, Sarath Chandar, Nan Rosemary Ke, et al. 2017a. A deep reinforcement learning chatbot. arXiv preprint arXiv:1709.02349.

Iulian V Serban, Alessandro Sordoni, Yoshua Bengio, Aaron Courville, and Joelle Pineau. 2016. Building end-to-end dialogue systems using generative hierarchical neural network models. In Thirtieth AAAI Conference on Artificial Intelligence.

Iulian Vlad Serban, Alessandro Sordoni, Ryan Lowe, Laurent Charlin, Joelle Pineau, Aaron Courville, and Yoshua Bengio. 2017b. A hierarchical latent variable encoder-decoder model for generating dialogues. In Thirty-First AAAI Conference on Artificial Intelligence.

Pararth Shah, Dilek Hakkani-Tur, Bing Liu, and Gokhan Tur. 2018. Bootstrapping a neural conversational agent with dialogue self-play, crowdsourcing and on-line reinforcement learning. In Proceedings of the 2018 Conference of the North American Chapter of the Association for Computational Linguistics. 
Human Language Technologies, Volume 3 (Industry Papers), pages 41-51.

Weiyan Shi and Zhou Yu. 2018. Sentiment adaptive end-to-end dialog systems. In Proceedings of the 56th Annual Meeting of the Association for Computational Linguistics (Volume 1: Long Papers), pages 1509-1519.

Jamin Shin, Peng Xu, Andrea Madotto, and Pascale Fung. 2019. Happybot: Generating empathetic dialogue responses by improving user experience lookahead. arXiv preprint arXiv:1906.08487.

Candace L Sidner, Cory D Kidd, Christopher Lee, and Neal Lesh. 2004. Where to look: a study of humanrobot engagement. In Proceedings of the 9 th international conference on Intelligent user interfaces, pages 78-84. ACM.

Robert F Stengel. 1986. Stochastic optimal control. John Wiley and Sons New York, New York.

Pei-Hao Su, Paweł Budzianowski, Stefan Ultes, Milica Gasic, and Steve Young. 2017. Sample-efficient actor-critic reinforcement learning with supervised data for dialogue management. In Proceedings of the 18th Annual SIGdial Meeting on Discourse and Dialogue, pages 147-157.

Philip Thomas and Emma Brunskill. 2016. Dataefficient off-policy policy evaluation for reinforcement learning. In International Conference on $\mathrm{Ma}$ chine Learning, pages 2139-2148.

Emanuel Todorov. 2007. Linearly-solvable markov decision problems. In Advances in neural information processing systems (NIPS), pages 1369-1376.

Hado Van Hasselt, Arthur Guez, and David Silver. 2016. Deep reinforcement learning with double qlearning. In Thirtieth AAAI Conference on Artificial Intelligence.

Harry Weger Jr, Gina R Castle, and Melissa C Emmett. 2010. Active listening in peer interviews: The influence of message paraphrasing on perceptions of listening skill. The Intl. Journal of Listening, 24(1):3449 .

Li Zhou, Jianfeng Gao, Di Li, and Heung-Yeung Shum. 2018. The design and implementation of xiaoice, an empathetic social chatbot. arXiv preprint arXiv:1812.08989.

Daniel M Ziegler, Nisan Stiennon, Jeffrey Wu, Tom B Brown, Alec Radford, Dario Amodei, Paul Christiano, and Geoffrey Irving. 2019. Fine-tuning language models from human preferences. arXiv preprint arXiv:1909.08593.

\section{A Reproducibility}

\section{A.1 Training details and hyperparameters}

\section{Baseline Models}

The underlying architecture of the baseline language models employed for this work is a Variational Hierarchical Recurrent Encoder Decoder (VHRED) (Serban et al., 2017b). We also conduct a second set of experiments on an enhanced version of this model with additional knowledge distillation to improve the model's ability to track the sentiment and semantics of the conversation, as proposed by Ghandeharioun et al. (2019). The language models were originally trained on two datasets: movie dialogs (Danescu-NiculescuMizil and Lee, 2011) and a dataset scraped from reddit.com/r/casual_conversation (Ghandeharioun et al., 2019).

The underlying parameters of the VHRED model were as follows: Context RNN hidden size $=1000$, decoder hidden size $=1250$, encoder hidden size $=1250, z$ embedding size $=600$, gradient clip $=1.0$, dropout $d=0.2$. The maximum conversation length was fixed at 5 utterances (context from more than 5 utterances ago was discarded), and the maximum sentence length was 30 tokens. The VHRED model has 76.6 million parameters.

We also added layers to the Context RNN and regularized it to be able to predict the semantic content of the input utterance using a form of knowledge distillation (Hinton et al., 2015) from a stateof-the-art sentence-embedding model (Conneau et al., 2017). There were 2 additional feedforward semantic prediction prediction layers of size 128 , which used ReLu activation. The VHRED model with sentiment and infersent regularization has 95.4 million parameters.

Each RL model was trained on a NVIDIA GeForce GTX 1080 GPU.

\section{RL Models}

The RL models, the main focus of our work, were trained using human conversation data collected via the online interactive platform (described in Section F) and batch size was fixed at 32. Each model was trained for 2000 epochs. The RL models were initialized with the weights of the best model trained on the Reddit dataset. Early stopping was used to determine the number of training iterations of the best checkpoint. For each bot, 3 different stopping epochs were tested and the best was selected. The checkpoint was selected using 
manual tuning based on interactive chat with the chatbots. For the best performing bots, KL-Control $Q$ and KL-Control $\Psi$, the 1600 and 1800 epoch checkpoints were selected respectively.

The reward weights were also tuned to determine which weighting of rewards produced the desired bot behavior. We tried uniform weights (summing up to 1) and slightly increased weights for repetition rewards and human bot interaction rewards. The best weights were found to be assigning 0.15 to repetition and human bot interaction rewards and 0.1 to all other rewards. Reward weights were also determined using manual tuning and conversational interaction. The same reward weights were shared between all RL models we trained. Only 3 sets of weights were tried in the reward weights hyperparameter optimization process.

All other hyperparameters were shared between RL models, and were as follows: discount $\gamma=0.5$, weight placed on RL reward vs. KL-divergence term $c=2$, number of Monte Carlo samples of the Target $Q$-network $M=5$, target network update rate $\alpha=.005$, learning rate $r=.0001$. We used a smooth $L 1$ loss function to approximate the $Q$ values, and clipped gradients at a value of 1.0. The RL models have a total of 76.6 parameters (same as the VHRED models).

\section{A.2 Computing Infrastructure}

Each RL model was trained on a NVIDIA GeForce GTX 1080 GPU. Training models for 2000 epochs took approximately 30 minutes for each model. The runtime for training the VHRED baseline models is around 6 hours. The speediness of training the RL models illustrates the scalability of RL training in improving dialog models for specific features.

\section{A.3 Model Validation and Evaluation}

We use interactive human evaluation through an online chat interface. Human participants are recruited using Amazon Mechanical Turk and rate either 7 or 8 bots each. Participants were instructed to continue the conversation through at least $6 \mathrm{hu}$ man responses. After the conversation, participants are asked to rate each bot in terms of Quality, Fluency, Diversity, Contingency, and Empathy on a 7-point Likert scale. A detailed example of the chat and interaction platform can be found in Section F. Since our models are evaluated using interactive chat, we also validate our models through interactive chat and rate the models while tuning hyperparameters. The authors interacted with and rated bots during to validate bots

\section{B Offline-RL with VHRED with Emotion and Infersent Regularization}

We also conducted experiments using each offline RL algorithm with a Sentiment and Infersent regularized VHRED Model. As described in Section A.1, by adding about 20 million extra parameters to the VHRED model in order to better achieve semantic coherence and sentiment contingency, the VHRED-EI (Emotion and Infersent regularized) model is a better performing baseline in terms of human ratings (Ghandeharioun et al., 2019).

We conducted the same human experiments where we recruited participants from Amazon Mechanical Turk to chat with and rate each dialog model. We found similar results as presented in our main paper. While our KL-control models achieved higher qualitative ratings than the other offline RL algorithms, none of the RL models received higher qualitative ratings than the VHRED-EI Model (Table 4). We also replicated training the KL-Control $\Psi$ model on single rewards and found that training on User Sentiment elicited the highest human qualitative ratings (Table 5). This consistent with our results on the VHRED model.

\section{Traditional RL experiments}

To demonstrate the effectiveness of these techniques, we tested them on traditional RL tasks using the OpenAI gym (Brockman et al., 2016), focusing on the CartPole-vO and Acrobot-v1 experiments. We first train an online $Q$-learning $B e$ havior policy, and store all $\left(s, a, r, s^{\prime}\right)$ experience samples into a replay buffer. We use this buffer to train a prior model of $p(a \mid s)$ using a Variational Auto-encoder. The VAE was trained to reconstruct the next state given the current state, $p\left(s^{\prime} \mid s\right)$, using a mean-squared error loss. The next action was predicted from the latent embedding $z$, meaning the model learned three functions: $z=f_{e}(s)$, $s^{\prime}=f_{d}(z)$, and $a=f_{a}(z)$. For Cartpole, both the encoder and decoder were made up of two linear layers with 750 neurons each. The latent dimension of the VAE was size 256. For Acrobot, the encoder and decoder had only one layer of size 256 each, and the latent dimension was 64 .

This VAE is used as a part of both the DBCQ and WOP algorithms. We can also use it for imitation learning, by sampling actions directly from $p(a \mid s)$ to obtain Behavioral Cloning (BC). We 


\begin{tabular}{|l|lllll|l|r|r|}
\hline Model type & \multirow{2}{*}{ Quality } & Fluent & Diverse & Related & Empathy & Total & Votes & $\begin{array}{l}\text { Human } \\
\text { reward }\end{array}$ \\
\hline VHRED-EI Baseline & $3.11 \pm .41$ & $4.34 \pm .44$ & $\mathbf{4 . 6 6} \pm . \mathbf{4 9}$ & $3.02 \pm .47$ & $3.45 \pm .47$ & $18.59 \pm 1.76$ & 0.19 & -0.05 \\
\hline DBCQ & $1.64 \pm .48$ & $1.87 \pm .34$ & $\mathbf{3 . 1 3} \pm . \mathbf{5 8}$ & $1.84 \pm .34$ & $2.09 \pm .38$ & $10.58 \pm 1.55$ & -0.23 & -0.02 \\
Batch $Q$ & $1.87 \pm .30$ & $2.36 \pm .42$ & $2.20 \pm .41$ & $1.91 \pm .32$ & $2.58 \pm .47$ & $11.91 \pm 1.58$ & -0.16 & 0.00 \\
Batch $Q+$ MC & $1.85 \pm .39$ & $2.46 \pm .44$ & $2.46 \pm .52$ & $1.98 \pm .39$ & $2.34 \pm .49$ & $11.07 \pm 1.82$ & -0.07 & $\mathbf{0 . 0 3}$ \\
KL-control $Q$ & $\mathbf{2 . 3 8} \pm .39$ & $3.24 \pm .47$ & $3.42 \pm .54$ & $\mathbf{2 . 3 8} \pm .45$ & $2.56 \pm .43$ & $13.98 \pm 1.81$ & 0.02 & 0.01 \\
KL-control $\Psi$ (WOP) & $2.33 \pm .41$ & $\mathbf{3 . 7 3} \pm . \mathbf{. 3 3}$ & $2.82 \pm .50$ & $2.31 \pm .44$ & $\mathbf{3 . 4 7} \pm . \mathbf{5 0}$ & $\mathbf{1 4 . 6 7} \pm \mathbf{1 . 8 2}$ & $\mathbf{0 . 1 3}$ & $\mathbf{0 . 0 3}$ \\
\hline
\end{tabular}

Table 4: Interactive human evaluation of offline RL techniques on the VHRED-EI Model. Ratings are Likert scale with $95 \%$ confidence interval $(n=45)$, votes and human reward are $z$-scores.

\begin{tabular}{|l|lllll|l|r|r|}
\hline $\begin{array}{l}\text { Reward } \\
\text { function }\end{array}$ & Quality & Fluent & Diverse & Related & Empathy & Total & Votes & $\begin{array}{l}\text { Human } \\
\text { reward }\end{array}$ \\
\hline Conv. len. & $2.20 \pm .40$ & $3.61 \pm .53$ & $3.02 \pm .52$ & $2.25 \pm .46$ & $2.48 \pm .45$ & $13.57 \pm 1.84$ & -0.04 & -0.01 \\
Infersent Coher. & $1.93 \pm .34$ & $3.50 \pm .45$ & $2.37 \pm .45$ & $2.11 \pm .45$ & $2.52 \pm .48$ & $12.43 \pm 1.75$ & -0.02 & -0.01 \\
User laughter & $1.96 \pm .38$ & $3.56 \pm .48$ & $2.33 \pm .51$ & $1.93 \pm .42$ & $3.20 \pm .55$ & $12.98 \pm 1.60$ & -0.15 & -0.01 \\
User Word Len & $2.11 \pm .32$ & $3.96 \pm .44$ & $3.04 \pm .45$ & $2.04 \pm .35$ & $2.55 \pm .46$ & $13.70 \pm 1.44$ & 0.06 & $\mathbf{0 . 0 4}$ \\
Manual votes & $2.14 \pm .38$ & $3.47 \pm .45$ & $2.91 \pm .47$ & $2.07 \pm .39$ & $2.42 \pm .46$ & $13.00 \pm 1.65$ & -0.03 & 0.01 \\
Sent. trans. & $2.02 \pm .31$ & $3.71 \pm .49$ & $2.98 \pm .50$ & $2.04 \pm .42$ & $2.84 \pm .48$ & $13.60 \pm 1.63$ & 0.03 & 0.01 \\
Bot Question & $2.29 \pm .37$ & $\mathbf{4 . 3 1} \pm . \mathbf{5 0}$ & $\mathbf{3 . 3 1} \pm . \mathbf{5 2}$ & $2.20 \pm .40$ & $2.60 \pm .41$ & $14.71 \pm 1.63$ & 0.06 & $\mathbf{0 . 0 4}$ \\
User Sentiment & $\mathbf{2 . 4 7} \pm .32$ & $4.05 \pm .45$ & $3.23 \pm .46$ & $\mathbf{2 . 4 2} \pm .39$ & $\mathbf{3 . 2 3} \pm . \mathbf{5 5}$ & $\mathbf{1 5 . 4 0} \pm \mathbf{1 . 4 9}$ & $\mathbf{0 . 0 9}$ & $\mathbf{0 . 0 4}$ \\
\hline
\end{tabular}

Table 5: Interactive human evaluation of WOP trained with different reward functions on VHRED-EI model. Ratings are Likert scale with $95 \%$ confidence interval $(n=45)$, votes and human reward are $z$-scores.

benchmark all of these techniques against vanilla $Q$-learning on the batch data (Batch $Q)$. All $Q$ networks shared the same underlying architecture: three fully-connected layers of size [256, 128, 64], with ReLU activation between. All models were trained with the Adam optimizer (Kingma and $\mathrm{Ba}$, 2014).

For each experiment, we ran 50 trials of each model with a different random seed each time. The Behavior policy was trained for a total of 20,000 steps in the environment, so in the Full buffer condition offline agents saw 20,000 experience samples. The Behavior policy typically converged before 10,000 steps, so in the Expert demonstrator condition the offline agents received the last 10,000 experience samples from the trained agent. In the Concurrent condition, offline agents saw a moving window of 1000 samples, since the online learner only used the most recent 1000 samples in the buffer for learning. The learning rate was .001, $\gamma=.99$, and $\epsilon$ decayed linearly from 1.0 to .01 over 2000 steps. The KL-constraint was computed as $D_{K L}[q(\tau)|| p(\tau)]=\alpha \log p(a \mid s)-\beta \log \pi(a \mid s)$, where $\alpha=0.5$ and $\beta=0.1$. DBCQ sampled $n=2$ actions before selecting the best action based on the maximum $Q$-value; note that in this environment there are only 2 actions. For Cartpole we used the $\Psi$-learning loss, and for Acrobot we used the traditional $Q$-learning loss.

We experiment with four different conditions which vary the quality of the Behavior policy and the replay buffer data: a) Full buffer: all experience samples experienced during online training are used for offline learning; b) Concurrent: the offline learning algorithms see a sliding window of experience samples in the same order that the online learner experienced them; c) Expert demonstrator: the buffer only contains experience generated by a fully trained online learner; and d) Noisy demonstrator: the online learner has a high probability of acting randomly $(\epsilon=0.3)$ and is thus a bad model of the optimal policy.

Figure 4 shows the results. Across conditions, we see that WOP is able to outperform Batch $Q$, imitation learning (BC), DBCQ, and the original behavior policy. As expected, Imitation learning (BC) underperforms other techniques when the batch contains noisy or inexpert experience samples. However, when the batch contains only expert trajectories, Batch $Q$ fails to learn, because the batch does not cover the full state-action space well, increasing extrapolation error. DBCQ matches or outperforms $\mathrm{BC}$ and Batch $Q$ in all scenarios. However, because DBCQ acts by sampling from $p(a \mid s)$ as learned by the $\mathrm{BC}$ model, its performance suffers when the batch data is noisy or imperfect. In 


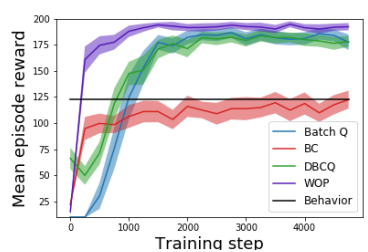

(a) Full buffer

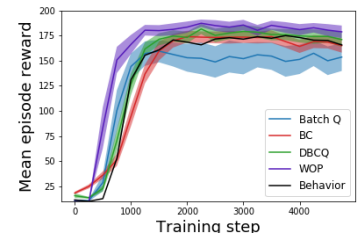

(b) Concurrent

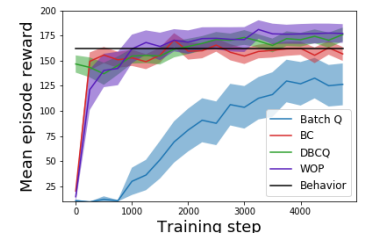

(c) Expert demonstrator

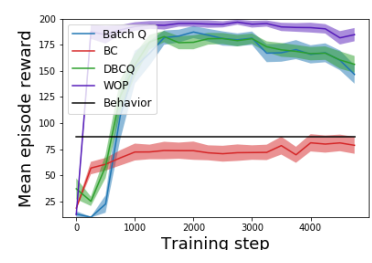

(d) Noisy demonstrator

Figure 4: Comparison of batch RL algorithms in Cartpole-v0 for different offline learning conditions. WOP consistently exceeds the performance of Batch Q-learning, Behavioral Cloning (BC), DBCQ, and the Behavior policy used to generate the batch data. Error bars show $95 \%$ CI of the mean over 50 trials.

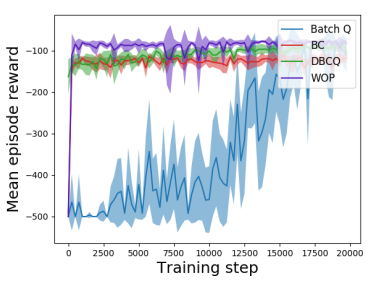

(a) Full buffer

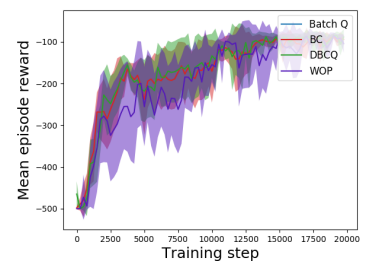

(b) Concurrent

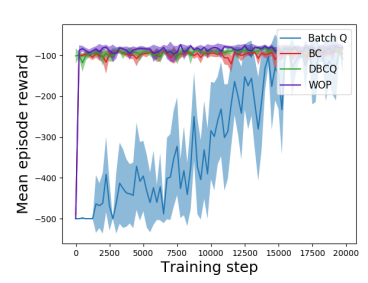

(c) Expert demonstrator

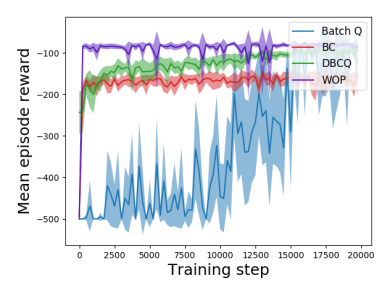

(d) Noisy demonstrator

Figure 5: Comparison of batch RL algorithms for different offline learning conditions in Acrobot-v1.

contrast, WOP is able to learn to trade-off staying close to the prior and obtaining higher reward, and consistently outperforms all other algorithms in this environment.

\section{Additional results}

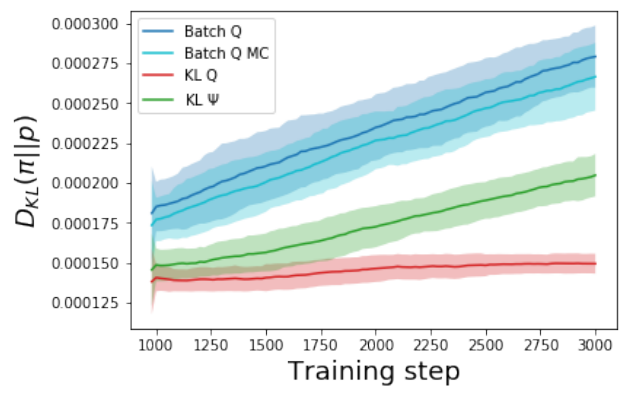

Figure 6: KL-divergence of the policy from the prior is lower with KL-control throughout training. Bands show $\sigma$.

Figure 6 shows the KL-divergence between RL policies and the prior language model throughout offline RL training. Without KL-regularization, the baseline RL models diverge quickly and continuously from the prior, losing information about realistic sequences. This figure also helps explain the poor performance of DBCQ in Table 2. The underlying $Q$-network in DBCQ does not directly integrate the prior. As $Q$-learning causes the model to diverge from the prior, the $Q$-estimates of lan- guage generated according to the prior become unrealistic, and selects unrealistic actions. This results in highly 'diverse' (random) generated utterances. Note that since we operate in discrete action space, we could not include the perturbation model originally proposed by (Fujimoto et al., 2018), which may be critical to achieving good performance with BCQ.

\section{E Implicit Rewards Details}

The total reward used to train the bots is a combination of the rewards described in Table 6 . These rewards were selected based on the average z-score of rewards for utterances that were upvoted and downvoted. Figure 8 shows all the user rewards and that User Laughter and User Sentiment reward scores correlate with upvotes and downvotes. Figure 9 shows all the bot rewards with Bot Sentiment, Bot Laughter, Bot Convo. Repetition, and Bot Utterance Repetition as rewards that correlate with manual votes. Figure 10 shows the bot-user combined rewards, and that Word Similarity and USE Similarity are the rewards that correlate with manual up and downvotes.

\section{E.1 Sentiment-based}

To compute sentiment on short texts like conversation utterances, we leverage a state-of-the-art sentiment-detection model, which was trained on a massive amount of Twitter data to predict the emo- 


\begin{tabular}{|l|l|}
\hline Reward & Weight \\
\hline User Sentiment & 0.10 \\
User Laughter & 0.10 \\
USE Similarity & 0.15 \\
Word Similarity & 0.15 \\
Bot Question & 0.10 \\
Bot Sentiment & 0.10 \\
Bot Conversation Repetition & 0.15 \\
Bot Utterance Repetition & 0.15 \\
\hline
\end{tabular}

Table 6: Reward weights used for RL model training

jis in tweets (Felbo et al., 2017). Transfer learning from this model to other tasks showed that it was able to significantly outperform a series of sentiment, irony, and sarcasm benchmarks. This DeepMoji model outputs a probability distribution over 64 most-frequently used emojis as shown in Figure 7. After observing the performance of the model in detecting users' emotions in the domain of online chat, we define a set of weights over the emojis and calculate the weighted sum over an emotion embedding vector to derive a Sentiment reward which is higher for positive sentiment and lower for negative sentiment. These weights are shown in Figure 7 (b). We also compute a sentiment-transition reward using the same score based on whether the peak positive sentiment occurred later in the conversation than the peak negative sentiment, reasoning that sentiment should improve over the course of the conversation. The Bot Sentiment reward is the DeepMoji sentiment computed on the bot response, User Sentiment reward is the value computed on the user response, and the Sentiment Coherence reward is based on the similarly of user and bot sentiments.

\section{E.2 Engagement-based}

Based on prior work (Zhou et al., 2018), we use the number of turns in the conversation as an indicator of the quality of the bot's performance. To distribute this reward over every utterance in the conversation, we take the total conversation length $N$, and compute the discounted reward for utterance $n<N$ as $\gamma^{N-n} N$ (Conversation Length). We also reward each utterance with the number of words and characters in the user's response, which we refer to as User Ans. Word Len and User Ans. Char Len. We also examine how long bot responses are with the Bot Response Length reward.

\section{E.3 Laughter}

Laughter has been shown to be very important to human affiliation (Provine, 1996) and solidarity (Hay, 2000). Therefore, we detect the number of occurrences of strings indicating laughter (e.g. 'ha', 'lol') in the user's response, and use this as a reward. Interestingly, we find that bots trained to maximize user laughter learn to be extremely supportive and cheerful compared to other bots (for definitions of supportive and cheerful see section E.6).

\section{E.4 Semantic similarity}

Language style matching has been shown to be a strong predictor of relationship initiation and stability (Ireland et al., 2011). While it would be ideal if our chatbots could intelligently adapt their conversation style to a new user, in reality most baseline dialog models struggle to maintain topic coherence, even over a few utterances (for an analysis of this effect, see (Ghandeharioun et al., 2019)). Therefore we reward semantic similarity between the user's input and the bot's response, to encourage the bot to stay on topic and produce reasonable answers. The Infersent Cornell Coherence and Infersent Reddit Coherence rewards are computed using a sentence embedding model trained on the Reddit and Cornell corpora respectively (described in section A.1). We use the Universal Sentence Encoder ((Conneau et al., 2017)) to compute the USE Similarity reward. We also directly compute word overlap as a reward as Word Similarity.

\section{E.5 Questions}

Asking questions is an important listening skill, and is linked to conversation management, attentiveness, and responsiveness (Bodie et al., 2012). Therefore, we give the bot a reward of 0.5 if the utterance contains a question word (how, what, where, why, when, who), and an additional 0.5 if it contains a question mark. We refer to this reward as Bot Question.

\section{E.6 Phrase based rewards}

After training the bots on these rewards, we noticed a shift in the distribution of their language towards more polite, cheerful, and supportive speech. Therefore, we designed post-hoc metrics to measure these qualities, which are based on counting whether a subset of phrases is present in an utterance.

Compliment phrases: you are beautiful, you 


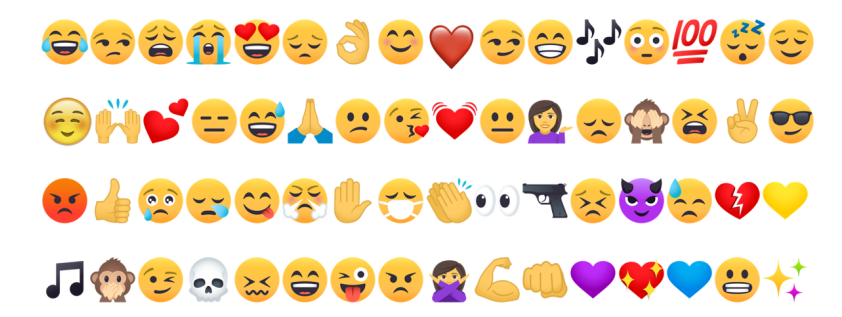

(a)

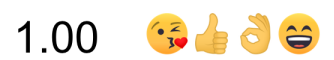

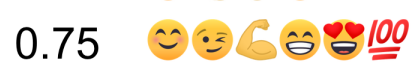

0.50 - $8=0.00000$

$-0.50=\cdots=a^{2 z} \cdot 0$

$-0.75 \% \because 20$

$-1.00$

(b)

Figure 7: (a) 64-most frequent emojis as predicted by (Felbo et al., 2017) used for calculating emotion embeddings. (b) Assigned weights used in producing the sentiment reward from the predicted emoji values.

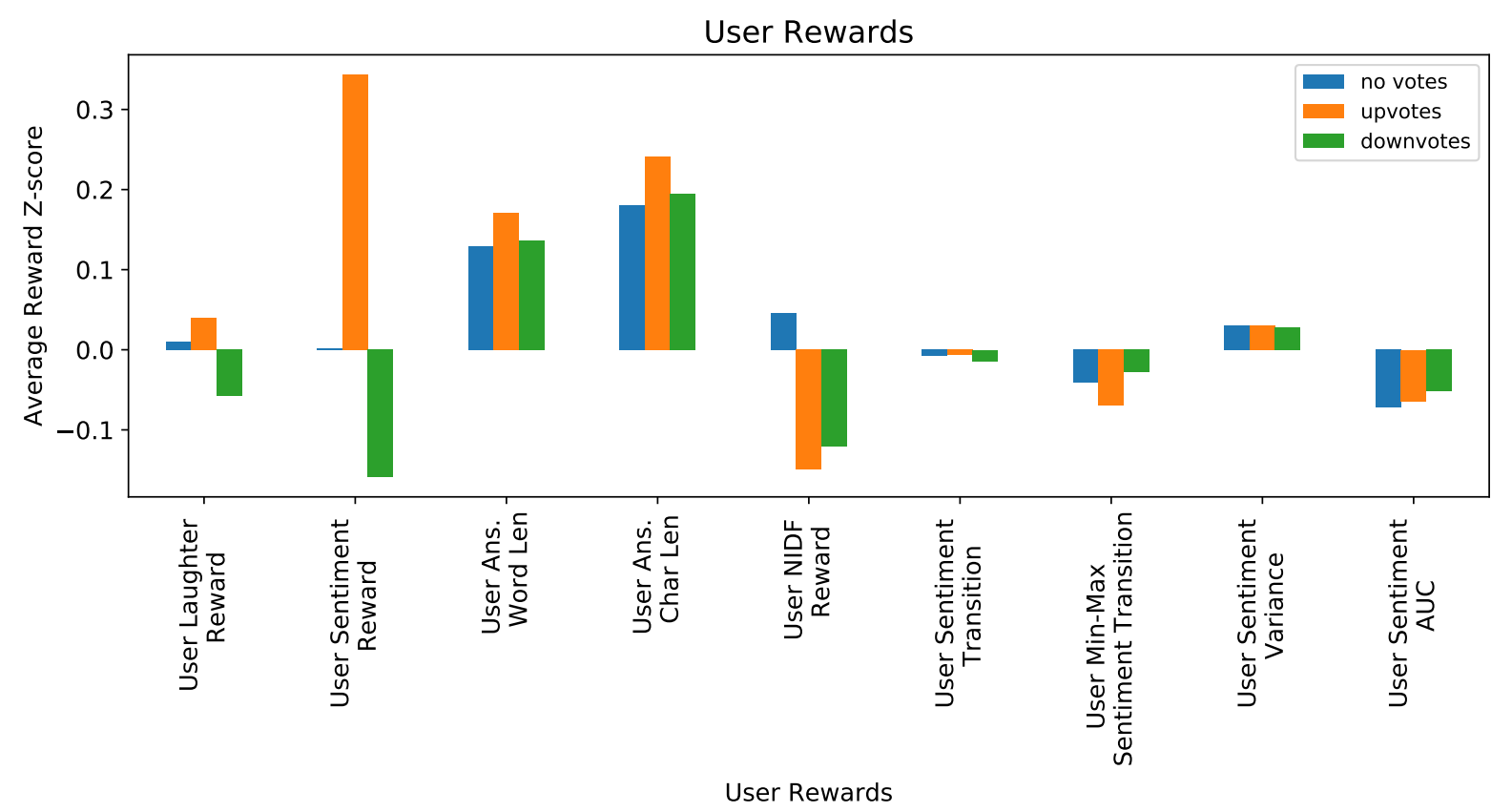

Figure 8: Mean z-scores for user-response-based rewards by manual vote

are so beautiful, you're beautiful, you're beautiful, you are the best, you're the best, i like you, you're a good, you re a good, i love the way you

Politeness phrases: if I may; may I; please; thanks; no worries; if you don't mind; have a great day; I'm sorry.

Supportive phrases: you're right; you are right; you're not alone; you are not alone; congrats; that's a good idea; that is a good idea; you'll be fine; you will be fine; you'll be okay; you will be okay; it will get better; sorry you're going through; sorry you are going through; if it makes you feel better; if it makes you feel any better; keep your head up; keep it up; I'm in a similar situation; I am in a similar situation; you'll get it; you will get it; happy for you; I'm in the same boat; I am in the same boat; if you feel like you need to vent.

Cheerful phrases: nice to hear; happy; excited; really nice; glad; the best; great; good time; looking forward; beautiful.

\section{E.7 Toxicity}

We also want to discourage our bot from malicious or offensive language. Saleh et al. (2019) incorporate a Toxicity Classifier trained with data from the Toxic Comment Classification Challenge ${ }^{3}$ as a reward in the training hierarchical RL dialog models. We compute Toxicity reward scores using this classifier as Bot Toxicity (e.g. lower toxicity score, higher Bot toxicity reward).

\section{E.8 Specificity}

Specificity within a conversation is valuable in avoid exchanging vacuous phrases back and forth.

\footnotetext{
${ }^{3}$ https://www.kaggle.com/c/ jigsaw-toxic-comment-classification-challenge
} 


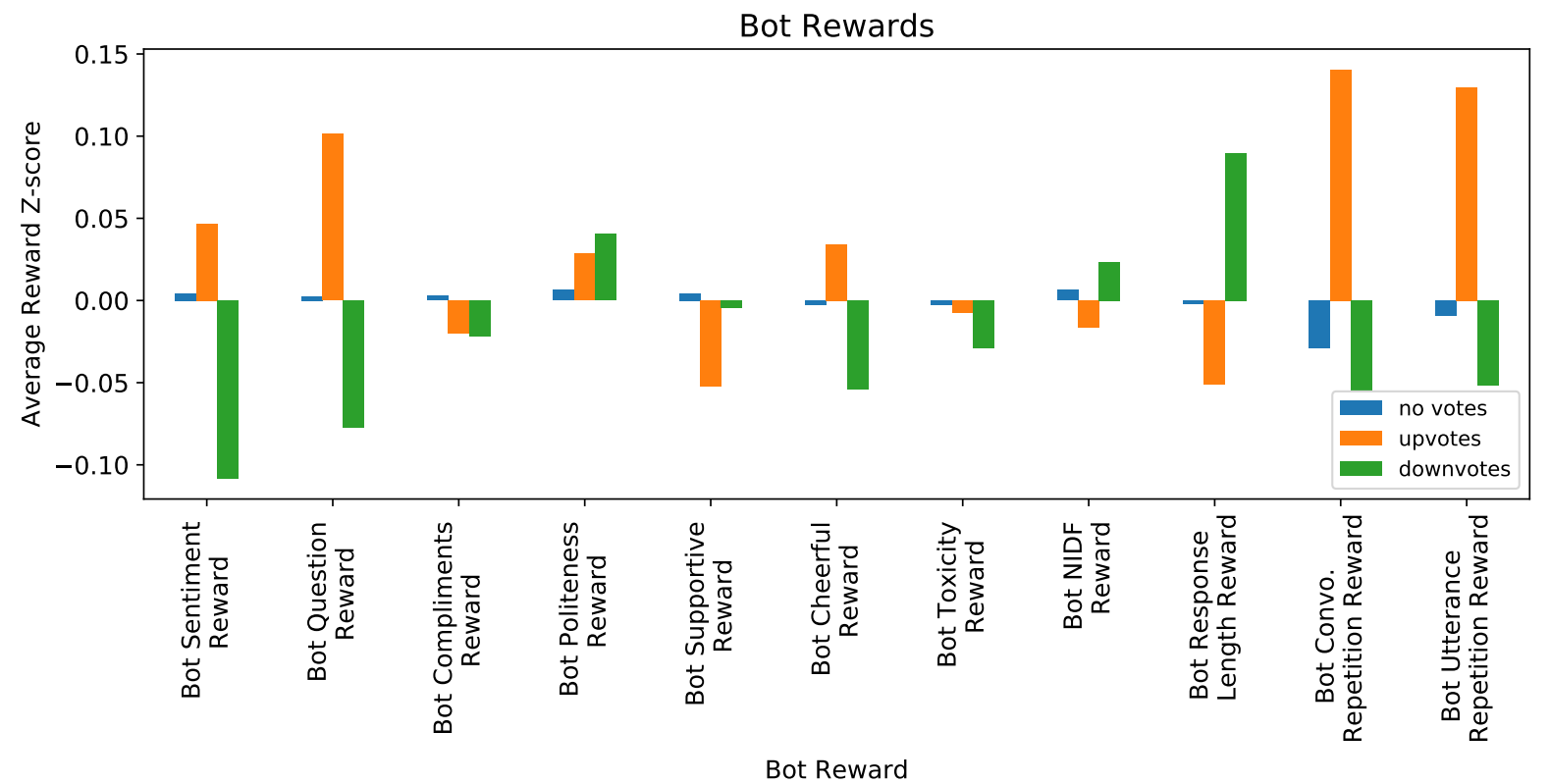

Figure 9: Mean z-scores for bot-based rewards by manual vote

However building a chit-chat bot without a knowledge graph back-end limits the level of substance that can be incorporated into a conversation. We use the approach from (See et al., 2019) of computing normalize IDF to create more specificity in the conversation. We compute NIDF on both user (User NIDF) and bot (Bot NIDF) text.

\section{E.9 Repetition}

While minimizing repetition is a common implicit goal of dialog systems, we will explicitly optimize for reducing repetition through repetition rewards. We compute utterance repetition by the number of non-unique words in each utterance as Bot Utterance Repetition Reward. We compute conversation repetition by the number of non-unique words in each conversation as Bot Convo. Repetition Reward. These rewards are negated since we want a higher reward score for less repetition. We also remove stop words in the computation of non-unique words.

\section{F Interactive bot platform details}

To collect data from humans interacting with our bots, we built a platform for hosting deep neural network dialog models online on GPU for fast, realtime inference. Figure 11 shows an example of the interface, in which users are able to rate the bots after talking to them for at least three turns.

Note that during the chat, annotators can optionally click the up and down arrows beside each chatbot response to give feedback on the specific utterance. Once 6 or more turns of the conversation has taken place, participants may click "Close Chat and Rate" to get to the rating screen.

We train our RL models based on chat data collected on this platform. Currently, the conversations contain Personally Identifiable Information such as user name, age, location, etc. We obtained for IRB approval for this study and cannot release the conversations at this time in their current form.

\section{F.1 Website server setup and configuration}

The server was hosted on a Google Cloud Platform virtual instance with 64GB of RAM and a NVIDIA Tesla P100 graphics card. The backend was a Django program being served by NGINX and uWSGI. For simplicity, we opted to have the Django process import the chatbots into the same Python process as Django, rather than have the two connect to each other via other means such as sockets. This configuration decreased development time and increased reliability, but it would need to be revisited if the server needed to scale several orders of magnitude past what was required for this study. The current configuration was still able to support hundreds of simultaneous users and host more than 30 bots concurrently.

The chatbots were kept in a separate project from the Django project and maintained separately from the server code. Each chatbot extended an abstract class that defined key methods for the Django pro- 


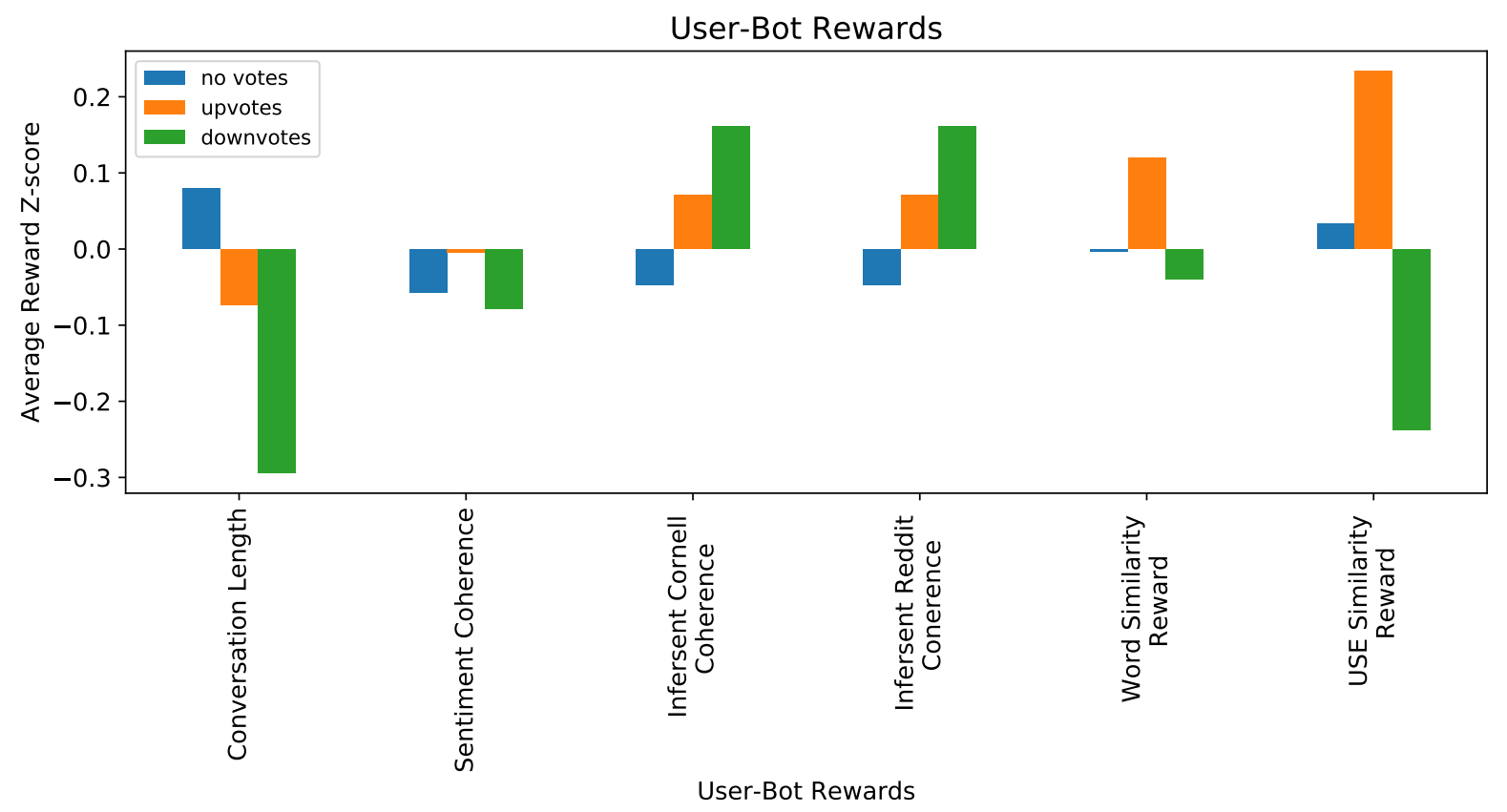

Figure 10: Mean z-scores for bot-user-based rewards by manual vote

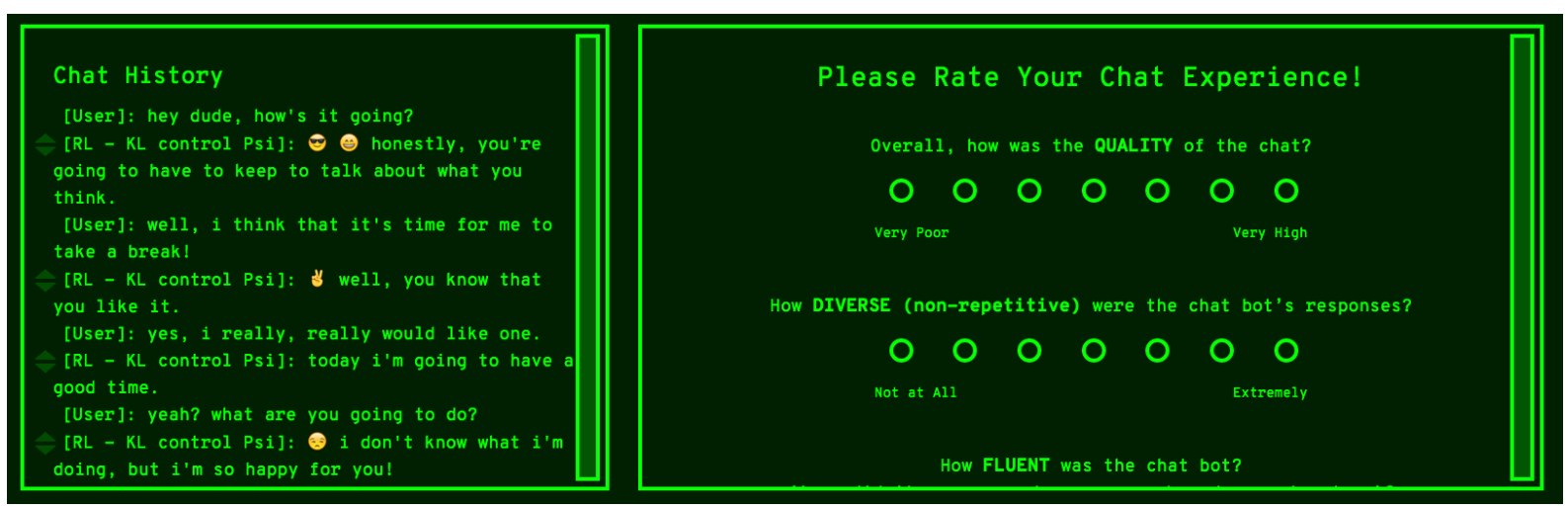

Figure 11: Interactive evaluation ratings page used to collect evaluations

gram to use, and was registered to a globally accessible dictionary via a decorator. The Django project was provided the path to the Chatbots project in its PYTHONPATH, so it could import the dictionary in which all the chatbot objects had been registered and use that to dynamically determine which chatbots were available and to access them in its views.

It is important to note that the chatbots used PyCUDA, and PyCUDA does not work in a multiprocessing environment. Because of this, uWSGI needed to be configured to only have one python process and to disable any attempt at multiprocessing. Furthermore, the chatbots required substantial startup times, so all chatbots are kept in memory at all times in the Django process. In order to keep all the chatbots in memory concurrently, we needed a very high amount of RAM on our server and opted for a $64 \mathrm{~GB}$ virtual instance, and a GPU with $16 \mathrm{~GB}$ RAM. This combination of CUDA to run the chatbots on the GPU with a high amount of RAM to keep all bots in memory at the same time resulted in incredibly fast server response times, with effectively no increase in response time when using the bots in requests compared to requests that did not.

For further information and instructions on server configuration, please read the server documentation available at https://github.com/ asmadotgh/neural_chat_web. We hope that this platform will allow others to host their own bots and evaluate them in an interactive setting. 